\author{
Martin A. Sieber \\ Philipp Lengsfeld \\ Thomas Frenzel \\ Sven Golfier \\ Heribert Schmitt-Willich \\ Fred Siegmund \\ Jakob Walter \\ Hanns-Joachim Weinmann \\ Hubertus Pietsch
}

\section{Preclinical investigation to compare different gadolinium-based contrast agents regarding their propensity to release gadolinium in vivo and to trigger nephrogenic systemic fibrosis-like lesions}

Received: 12 November 2007

Revised: 29 January 2008

Accepted: 7 March 2008

Published online: 11 June 2008

(C) The Author(s) 2008

M. A. Sieber $(\square)$. T. Frenzel

S. Golfier - H.-J. Weinmann - H. Pietsch

TRG Diagnostic Imaging,

Bayer Schering Pharma AG,

13353 Berlin, Germany

e-mail: martin.sieber@bayerhealthcare. com

Tel.: +49-30-46814439

Fax: +49-30-46894439

P. Lengsfeld

GMA DG Diagnostic Imaging,

Bayer Schering Pharma AG,

13353 Berlin, Germany

H. Schmitt-Willich

GDD Diagnostic Imaging,

Bayer Schering Pharma AG,

13353 Berlin, Germany
F. Siegmund · J. Walter

Nonclinical Drug Safety,

Bayer Schering Pharma AG,

13353 Berlin, Germany

\begin{abstract}
Recent reports suggest that nephrogenic systemic fibrosis (NSF) is associated with the administration of gadolinium (Gd)-based contrast agents (GBCAs) and in particular with the stability of the Gd-complex. The aim of this investigation was to compare GBCAs and their potential to trigger NSF. Forty-two healthy male rats received repeated intravenous injections of six different GBCAs at high doses to simulate the exposure seen in patients with severe renal dysfunction. Histopathological and immunohistochemical analysis of the skin was performed, and the concentrations of $\mathrm{Gd}$, zinc and copper were measured in several tissues by
\end{abstract}

inductive coupled plasma atomic emission spectroscopy. Macroscopic and histological skin changes similar to those seen in NSF patients were only observed in rats receiving Omniscan. In addition, very high concentrations of $\mathrm{Gd}$ were observed in the animals treated with Omniscan, and, to a lesser extent, in animals treated with OptiMARK. Significantly lower levels of $\mathrm{Gd}$ were found after the treatment with ionic linear agents and even less after the treatment with macrocyclic agents. The data in this investigation strongly suggest that the stability of the Gd-complex is a key factor for the development of NSFlike symptoms in this experimental setting.

Keywords Gadolinium • Nephrogenic systemic fibrosis . Contrast agents - Stability · Macrocyclic

\section{Introduction}

Nephrogenic systemic fibrosis (NSF) is a rare, but serious disorder that is characterized by fibrosis of the skin and of connective tissues throughout the body [1].

Although first described in the medical literature in 2000, the first reported case of NSF dates back to $1997^{1}$ [2]. Since then, the number of reports of NSF has increased with more than 250 reports of NSF filed with regulatory

\footnotetext{
${ }^{1}$ However, the actual term 'Nephrogenic Systemic Fibrosis' was introduced much later.
}

authorities worldwide as of August 2007 [3]. Although the exact etiology of NSF is currently unknown, it is most likely multifactorial and, to date has only been reported in patients with severe renal insufficiency [4], many of whom were on hemodialysis or peritoneal dialysis [5]. Recent case reports and data from animal experiments suggest an association between NSF and exposure to gadolinium (Gd)-based contrast agents (GBCAs) [6-8], although NSF without prior Gd exposure has also been reported [9, 10].

One theory suggests that the incidence of NSF is correlated to the stability of the various GBCAs [11]. The majority of cases reported to date relate to the use of one 
agent in particular, gadodiamide (Omniscan ${ }^{\circledR}$, Gd-DTPABMA), however, there are also reports with other agents such as gadoversetamide (OptiMark ${ }^{\circledR}$, Gd-DTPA-BMEA) and gadopentetate dimeglumine (Magnevist ${ }^{\mathbb{R}}$; Gd-DTPA). Up-to-date information on the number of reported cases for individual contrast agents as well as the regulatory status can be obtained on the websites of regulatory agencies such as the FDA [3].

In a previous investigation we have established a preclinical dosing regimen simulating the prolonged exposure to GBCAs seen in patients with severe renal impairment [8]. In that investigation a possible association between gadolinium exposure and the occurrence of NSFlike lesions in the investigated rats was established [8]. The aim of the present investigation is to further explore the differences between various marketed Gd-containing contrast agents regarding their potential to trigger NSF-like symptoms using the previously established experimental setting [8].

\section{Materials and methods}

Tested compounds

The tested Gd-containing contrast agents are all approved and marketed products and were purchased from their respective manufacturers. The following compounds were tested: Omniscan (gadodiamide, Gd-DTPA-BMA, GE Healthcare), OptiMARK (gadoversetamide, Gd-DTPABMEA, Covidien), Magnevist (gadopentetate dimeglumine, Gd-DTPA, Bayer Schering Pharma), MultiHance (gadobenate dimeglumine, Gd-BOPTA, Bracco Diagnostics), Gadovist (gadobutrol, Gd-BT-DO3A, Bayer Schering Pharma) and Dotarem (gadoterate meglumine, Gd-DOTA, Guerbet). ${ }^{2}$

\section{Animals}

Forty-two Hannover Wistar Rats [CRL:WI (GIx/BRL/ HAN)IGS BR] were obtained from Charles River (Sulzfeld, Germany), each weighing between 190 and 240 g. Six animals per group were selected at random and were housed with a 12-h day and night rhythm and were given water and standard diet (ssniff R/M-H from Sniff, Germany) ad libitum. Animals were treated according to the German Law on the Protection of Animals and with permission from the state animal welfare committee.

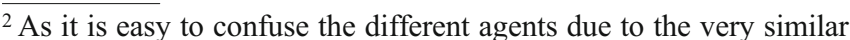
generic names and chemical abbreviations, as happened in the key publication by Grobner et al. [6], we have opted for using in this publication only the brand names as only the marketed formulations are investigated.
}

\section{Study design}

The investigation used the methodology previously established to simulate the high exposure to Gd-containing contrast agents in patients with severe renal impairment [8]. The formulations were injected into a tail vein daily, five times per week (not on weekends) at a dose of $2.5 \mathrm{mmol}$ $\mathrm{Gd} / \mathrm{kg}$ body weight over a period of 4 weeks. As the investigation control we used isotonic saline $(0.9 \%)$ solution (Table 1). The animals were sacrificed 5 days following the last treatment. This time point was chosen on the basis of serum elimination pharmacokinetics of the various compounds to ensure that the administered $\mathrm{Gd}$ compounds were completely eliminated from the body.

\section{Macroscopic skin findings, histology}

and immunohistochemistry

The animals were examined for macroscopic skin changes such as reddening, fur loss, scab formation and ulceration prior to every injection. At the end of the experiment the backs of the animals were shaved for better visualisation of the skin findings.

Immediately after necropsy, three skin probes were used to take skin samples from the back of each animal. The samples were taken from similar areas in each animal. The probes were fixed in $4 \%$ neutral buffered formalin. After fixation and routine dehydration, the tissue samples were embedded in paraffin and sectioned for hematoxylin-eosin and immunohistochemical staining. The histopathological reading was performed by two board-certified veterinary histopathologists (JW, FS) in a blinded fashion according to best practice guidelines of the Society of Toxicologic Pathology [12]. In general, structure and classification of the histopathology evaluation followed the logical framework described elsewhere $[13,14]$. The severity of the findings was graded using scores from 1 to 5 based on the increases in cellularity (namely increases in fibroblast-like

Table 1 Treatment groups and dosing

\begin{tabular}{lllll}
\hline Agent & $\begin{array}{l}\text { No. of } \\
\text { animals }\end{array}$ & Injections & $\begin{array}{l}\text { Dose per } \\
\text { injection } \\
(\mathrm{mmol} / \mathrm{kg})\end{array}$ & $\begin{array}{l}\text { Total dose } \\
(\mathrm{mmol} / \mathrm{kg})\end{array}$ \\
\hline Omniscan* & 6 & 20 & 2.5 & 50 \\
OptiMARK & 6 & 20 & 2.5 & 50 \\
Magnevist* & 6 & 20 & 2.5 & 50 \\
MultiHance & 6 & 20 & 2.5 & 50 \\
Dotarem & 6 & 20 & 2.5 & 50 \\
Gadovist & 6 & 20 & 2.5 & 50 \\
Saline & 6 & 20 & - & - \\
\hline
\end{tabular}

*Presented already in first publication [8] 
cells, dendritic-like cells and lymphocytes $)(1=$ minimal, $2=$ slight, $3=$ moderate, $4=$ marked, $5=$ massive).

Furthermore we performed CD34 stains of the dermis with the same methodology as described previously [8].

\section{Cell counts}

For the cell count in the skin, paraffin sections were dewaxed, rehydrated and stained with 4',6-diamidino-2phenylindole dihydrochloride (DAPI) (Sigma, St. Louis, MO, USA). Three skin samples were taken from each animal; two sections per sample were stained and two regions of interest (ROIs) per section (i.e., 12 ROIs per animal) were counted with the help of a cell-count software programme (Cellprofiler; www.cellprofiler.org). The ROIs were placed in the upper dermis to avoid areas with hair follicles, glands and fat tissue.

Gadolinium, zinc and copper measurements

Measurements of the $\mathrm{Gd}, \mathrm{Zn}$ and $\mathrm{Cu}$ concentrations in the skin (samples taken from the back, next to the histology probes), the liver, and the femur were made by inductive coupled plasma atomic emission spectroscopy (ICP-AES, IRIS Advantage, Thermo) at wavelengths of 342.247 , 213.856 and $224.700 \mathrm{~nm}$, respectively [15]. It should be noted that this method cannot distinguish between chelated or unchelated metal ions, in particular chelated or unchelated Gd.

\section{Statistical evaluation}

Gadolinium measurements (excluding saline control animals, where no Gd was detected) were tested globally for differences in medians between at least two groups with the non-parametric Kruskal-Wallis test. In case of significance at a two-sided 5\% level of significance, pairwise comparisons were performed using the non-parametric multi-comparison Dunn's test to ensure an experimentwise two-sided level of significance of $5 \%$.

For the $\mathrm{Cu}$ and $\mathrm{Zn}$ concentrations, each treatment group was compared with the saline control, and a one-sided 5\% level of significance $(p \leq 0.05)$ was used to assess noninferiority to saline (Dunnett's test). The non-inferiority margin was chosen as the mean of the saline group minus three times the standard deviation.

\section{Results}

Macroscopic and microscopic findings, histology, and immunohistochemistry

Five days after the 20th injection, macroscopic changes of the skin were observed in four out of six animals in the Omniscan-treated group. (Table 2, Figs. 1 and 2). Those findings included ulceration and crust formation. No macroscopic changes were observed in any of the other treatment groups or in the saline control group (Figs. 1 and 2).

On the histological level, fibrosis, including collagen deposit, loss of the extracellular space, thickening of the dermis and increased cellularity were observed in five out of six animals treated with Omniscan. Furthermore, an increase in the number of CD34-positive cells in the skin of the animals showing fibrosis (animals treated with Omniscan) was observed (data not shown). No histopathological findings of any severity were noted for any of the other investigated agents. Figure 2 shows a representative image from each treatment group.

\section{Cell counts}

To quantify the increase in cellularity, we counted the numbers of cells in the dermis, excluding the cells

Table 2 Overview of the observed macroscopic and microscopic findings in the dermis 5 days after the last injection

\begin{tabular}{|c|c|c|c|c|c|}
\hline \multirow[b]{3}{*}{ Agent } & \multirow{3}{*}{$\begin{array}{l}\text { Macroscopic changes } \\
\text { No. of animals }\end{array}$} & \multicolumn{4}{|c|}{ Microscopic changes } \\
\hline & & \multicolumn{2}{|c|}{ Increased cellularity } & \multicolumn{2}{|c|}{ Fibrosis sclerosis } \\
\hline & & No. of animals & $\begin{array}{l}\text { Mean severity score } \\
\text { (affected animals) }\end{array}$ & No. of animals & $\begin{array}{l}\text { Mean severity score } \\
\text { (affected animals) }\end{array}$ \\
\hline Omniscan & $4 / 6$ & $5 / 6$ & 1 & $5 / 6$ & 1.4 \\
\hline OptiMARK & $0 / 6$ & $0 / 6$ & - & $0 / 6$ & - \\
\hline Magnevist & $0 / 6$ & $0 / 6$ & - & $0 / 6$ & - \\
\hline MultiHance & $0 / 5$ & $0 / 5$ & - & $0 / 5$ & - \\
\hline Dotarem & $0 / 6$ & $0 / 6$ & - & $0 / 6$ & - \\
\hline Gadovist & $0 / 6$ & $0 / 6$ & - & $0 / 6$ & - \\
\hline Saline & $0 / 6$ & $0 / 6$ & - & $0 / 6$ & - \\
\hline
\end{tabular}




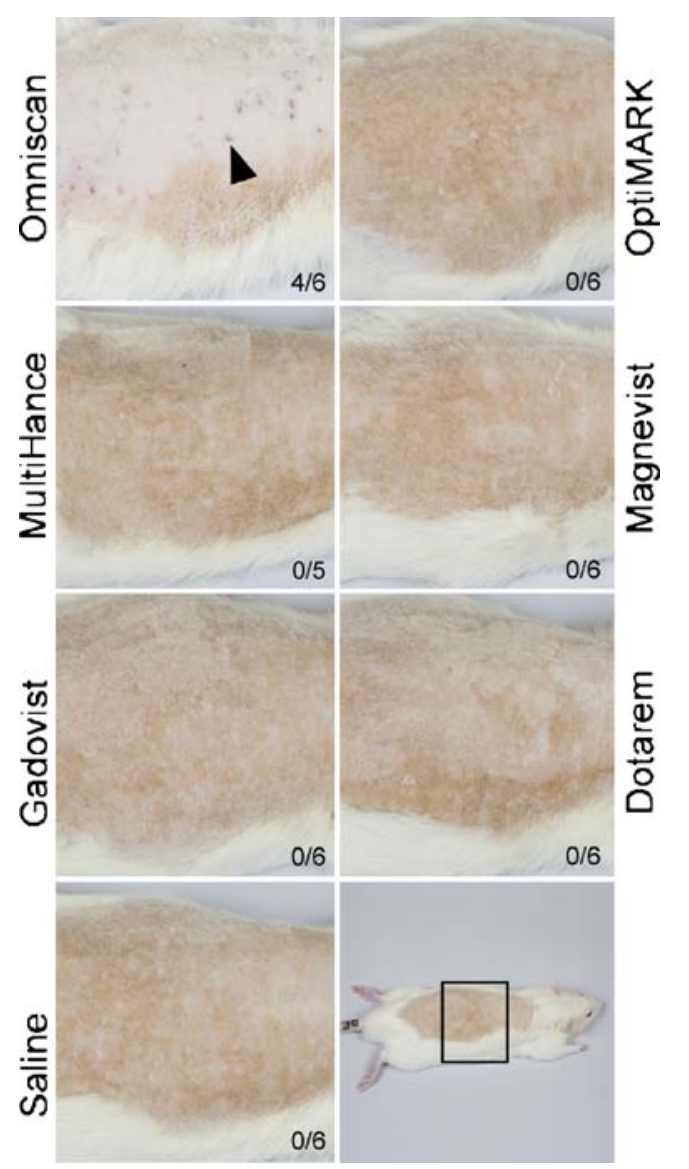

Fig. 1 Gross lesions in the skin of animals 5 days after the last injection of the indicated contrast media. Overview of the back of animals. One representative animal per group is shown. For better visibility of skin lesions, the animals were shaved (bottom right). The number of animals showing macrocopical skin lesions and the total number of animals is given. Please note that during the experiment one of the Multihance treated animals died. Skin lesions were observed only in animals treated with Omniscan (arrowhead)

associated with hair follicles, fat tissue and glands. We observed an increase in the number of cells in all the Omniscan-treated animals, which showed macroscopic changes of the skin (Fig. 3). No significant differences in cell counts were observed between the animals in the other treatments and the control group.

\section{Gadolinium levels in the skin}

Very high Gd concentrations were observed in skin samples from animals treated with Omniscan $(1.70 \pm 0.24 \mu \mathrm{mol} / \mathrm{g}$

Fig. 2 Fibrosing dermopathy in rat skin. Hematoxylin stain of skin probes taken 5 days after the last injection of the respective contrast media. Images $\mathbf{a}-\mathbf{g}$ (lefthand column) shown at $5 \times$ magnification, while images $\mathbf{h}-\mathbf{n}$ (righthand column) show $20 \times$ magnification of the dermis. Fibrosis of the dermis was observed in the Omniscantreated $(\mathbf{a}, \mathbf{h})$ animals only
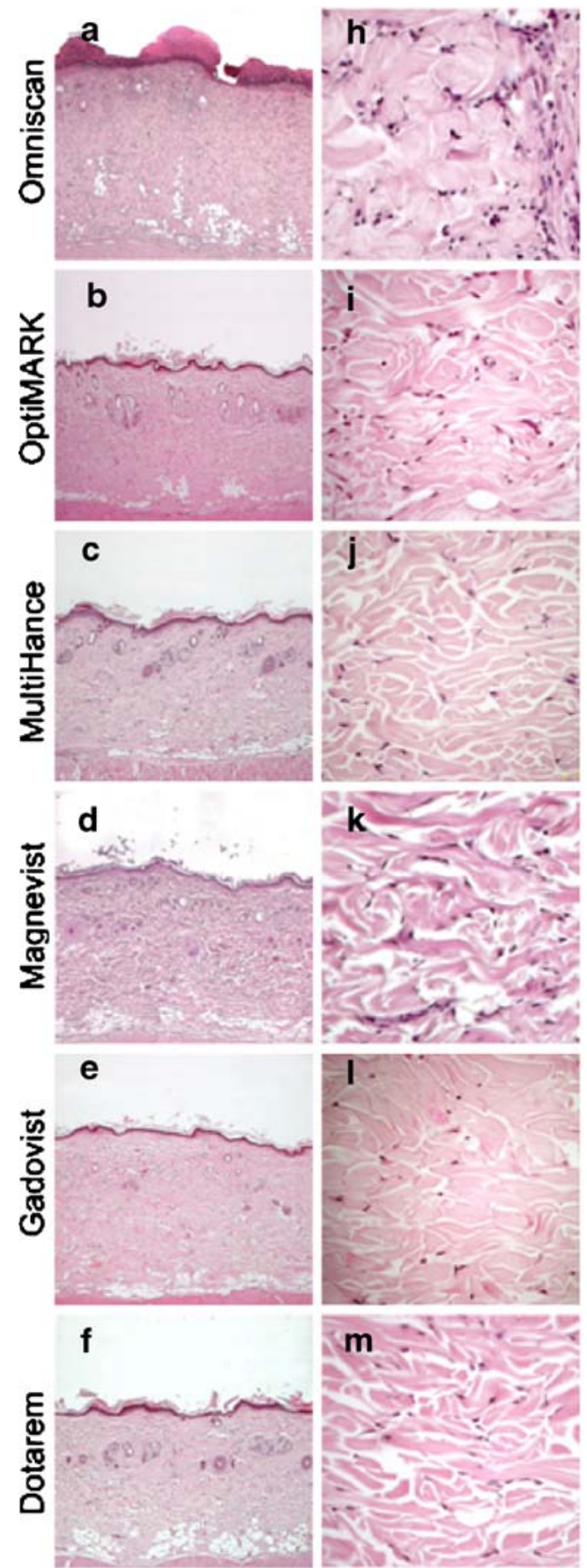

m

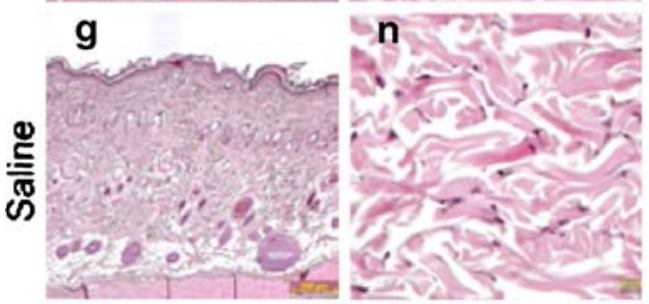


Fig. 3 Quantification of the increased cellularity. a Average number of cells observed in an individual animal treated with indicated contrast agents. b Nuclei (i.e. cells) are counted in paraffin sections stained with DAPI. c Average number of cells observed in treatment group. The number of cells is given per ROI. Asterisks indicate the animals with macroscopic skin changes. (DAPI4', 6-diamidino-2-phenylindole dihydrochloride, $R O I$ region of interest)
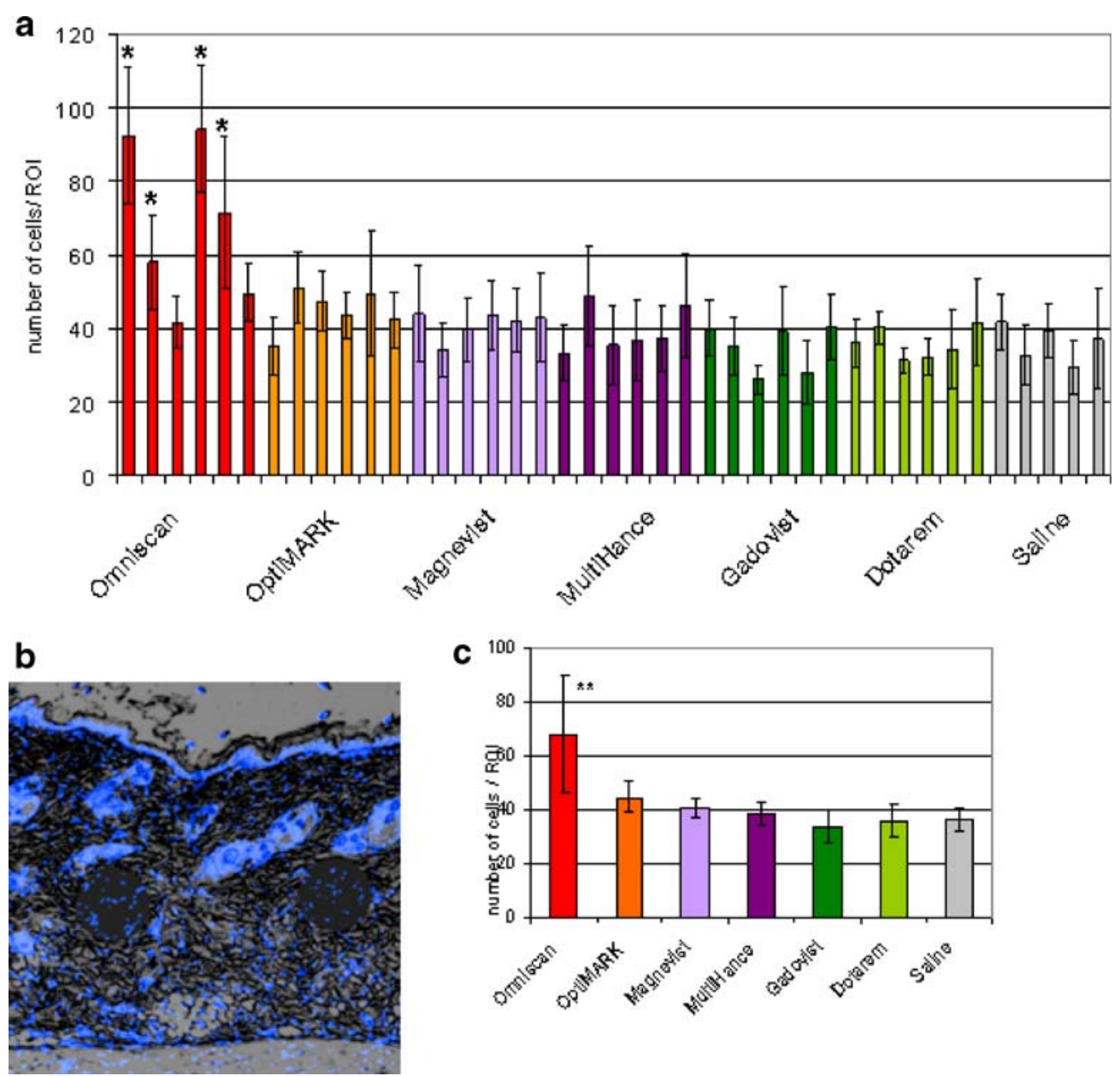

c

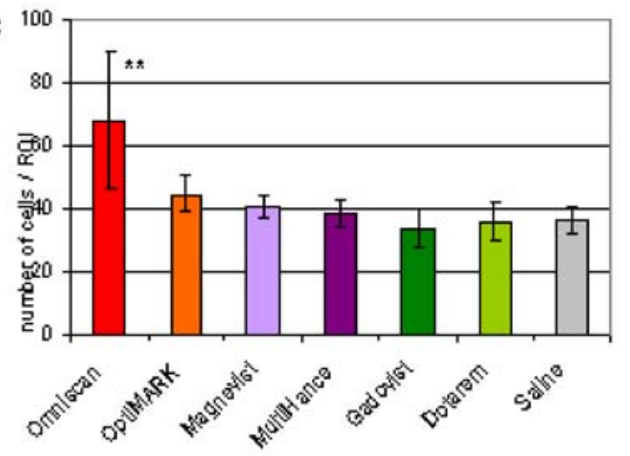

skin) (Fig. 4). The Gd concentration detected in the animals treated with Omniscan was almost 10-fold higher compared with the Gd concentration obtained following the administration of Magnevist and more than 30-fold higher compared to Gd concentrations detected following the administration of Gadovist and Dotarem. A global comparison of median Gd levels showed that the Omniscan treatment group had significantly higher Gd levels than all
Fig. 4 Gadolinium concentrations in the skin 5 days after the last injection of the indicated compound. Standard deviations are indicated by the error bars. Gd concentration after Omniscan administration was significantly higher compared to all other treatment groups $(*)$. MultiHance is excreted up to $50 \%$ hepatobiliarly in rats (\#). Gd concentrations after Dotarem and Gadovist were significantly lower than after administration of Omniscan, OptiMARK or Magnevist

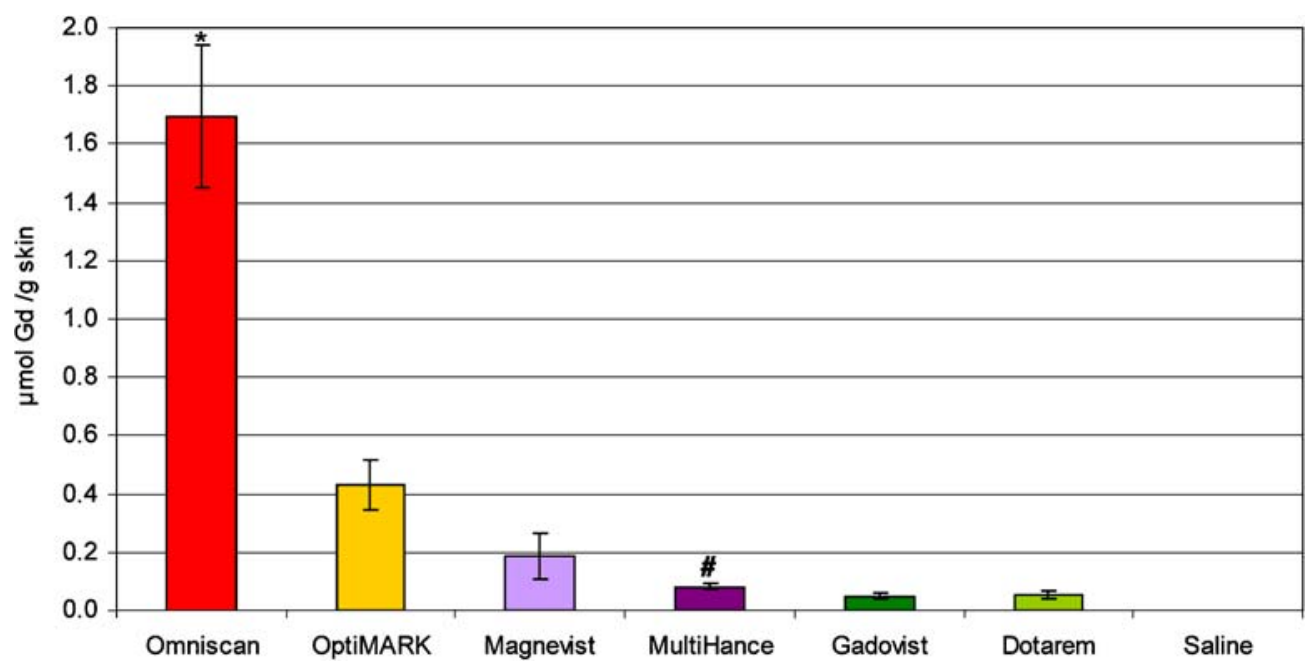


other treatment groups. A significantly lower Gd level was detected after application of OptiMARK $(0.43 \pm 0.09 \mu \mathrm{mol} /$ $\mathrm{g}$ skin), which contains twice the amount of excess ligand as compared to Omniscan. The Magnevist $(0.18 \pm 0.08 \mu \mathrm{mol} / \mathrm{g}$ skin) and MultiHance $(0.08 \pm 0.01 \mu \mathrm{mol} / \mathrm{g}$ skin) treatment groups also had significantly lower Gd levels than the Omniscan group. Gadolinium levels in the macrocyclic agents Gadovist $(0.05 \pm 0.01 \mu \mathrm{mol} / \mathrm{g}$ skin $)$ and Dotarem $(0.05 \pm 0.01 \mu \mathrm{mol} / \mathrm{g}$ skin $)$ treatment groups were the lowest of all investigated agents (Fig. 4).

Gadolinium levels in the liver and femur

In the liver and femur, the highest levels of Gd were again observed following treatment with Omniscan, with significant differences observed between Omniscan and all other treatment groups based on analyses of the median $\mathrm{Gd}$ levels (Fig. 5).
Zinc and copper levels in the skin, liver and femur

No significant loss of zinc and copper were observed for any of the treatment or control groups (Fig. 6).

\section{Discussion}

The possible association between the use of Gd-containing contrast agents in magnetic resonance imaging and the development of NSF in patients with severe renal impairment has been widely reported in the medical literature in recent months $[6,11,16]$. We initiated a range of preclinical in vivo studies to investigate this further. In an earlier investigation, a strong correlation was found between the gadolinium concentration in the skin and the development of NSF-like skin lesions in our preclinical setting [8]. The present investigation was performed to compare different marketed extracellular GBCAs in addition to the
Fig. 5 Gadolinium concentrations in the femur (a) and liver (b) 5 days after the last injection of the indicated compound. Standard deviations are indicated by the error bars. Gd concentration after Omniscan administration was significantly higher compared to all other treatment groups $(*)$. MultiHance is excreted up to $50 \%$ hepatobiliarly in rats (\#). In the femur Gd concentrations after Dotarem and Gadovist were significantly lower than after administration of Omniscan, OptiMARK or Magnevist
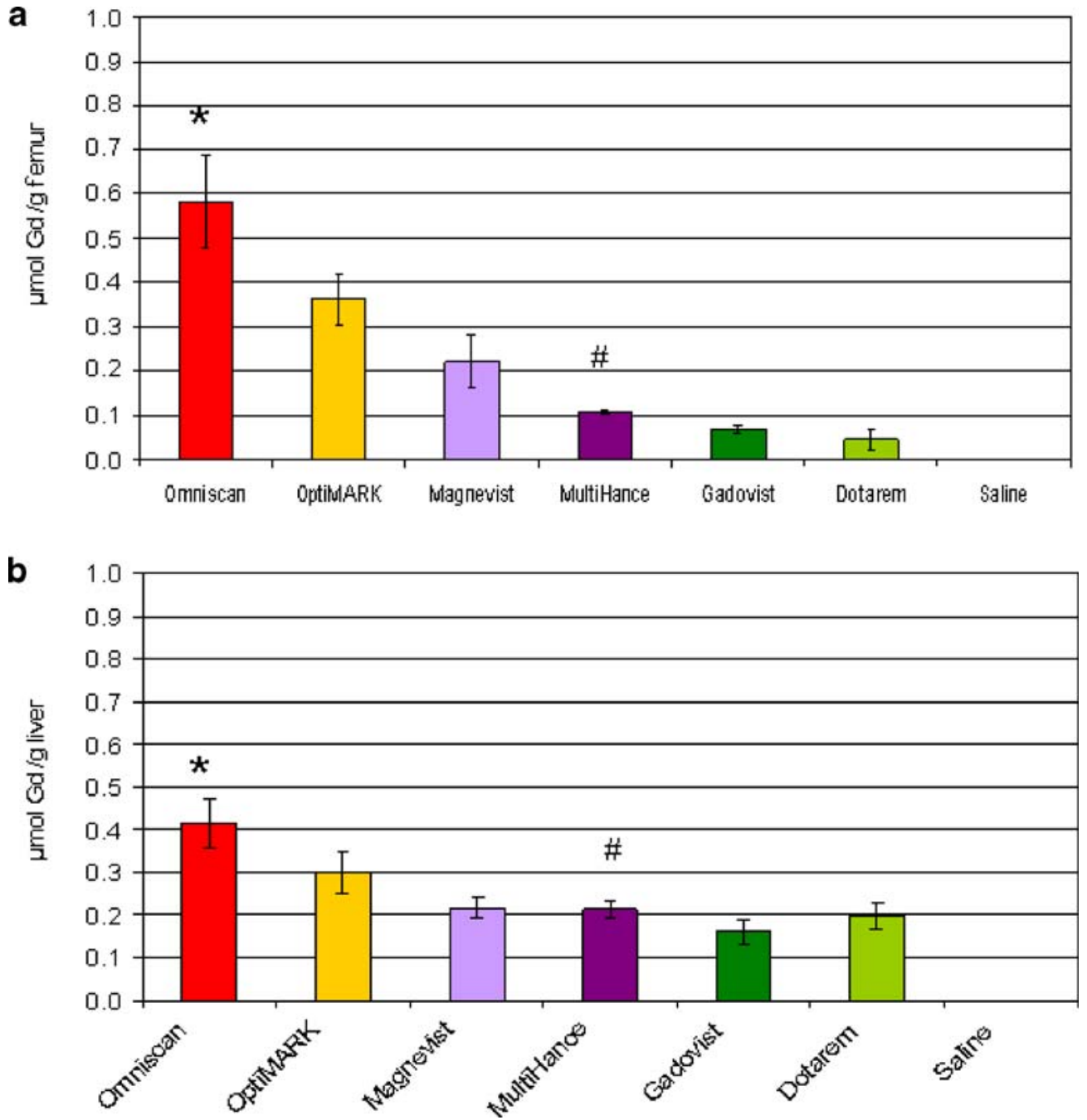
Fig. 6 Zinc (a) and copper (b) concentrations in the skin (purple), liver (yellow) and femur (green) 5 days after the last injection of the respective compound. Standard deviations are indicated
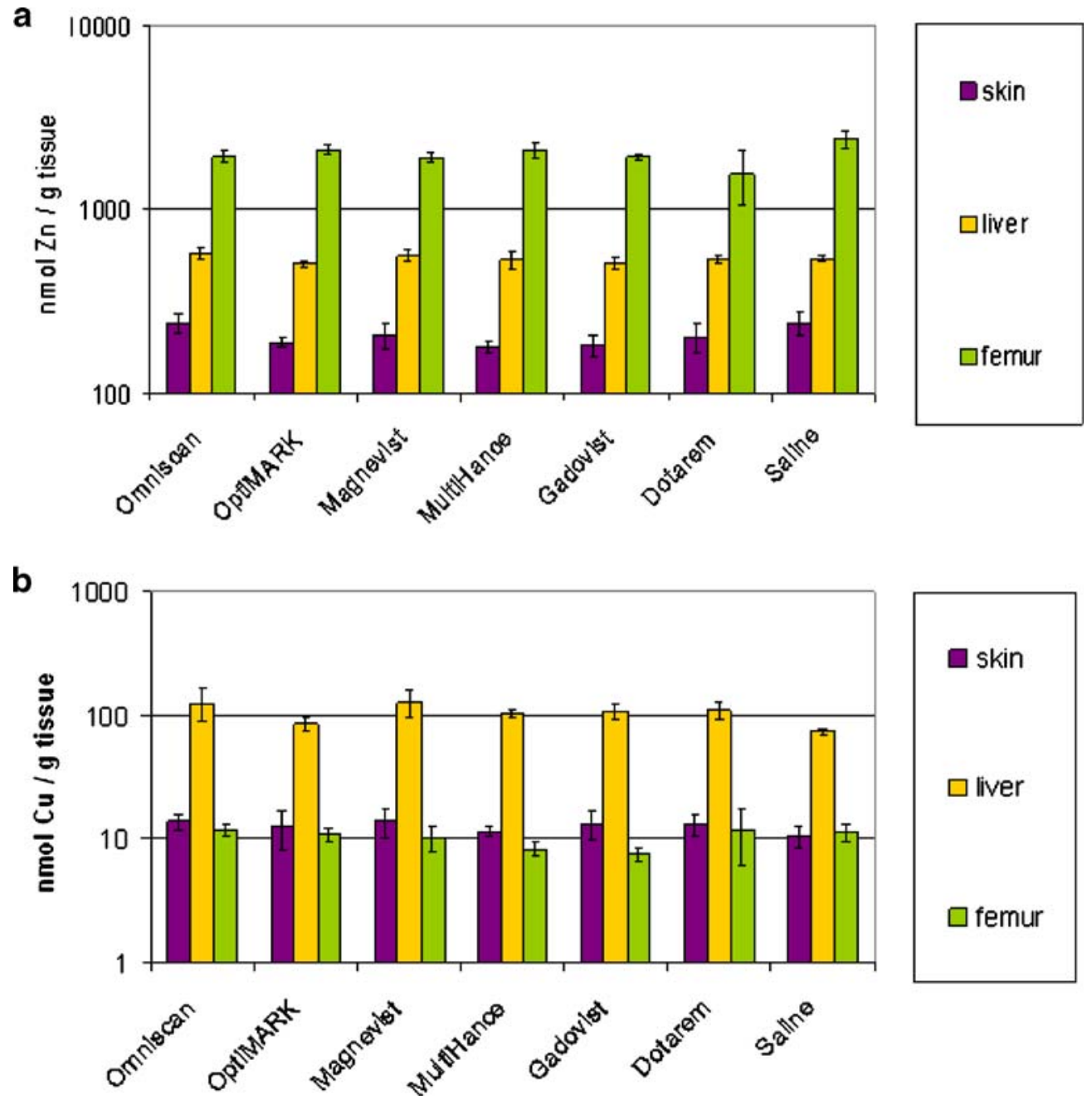

Magnevist and Omniscan groups already reported in the first publication [8].

After investigation of different key GBCAs in this setting, NSF-like skin lesions were only found in animals treated with Omniscan. In the skin of patients with NSF the lesions are accompanied by an increase in fibroblast-like cells, dermal macrophages and fibrocytes positive for CD34 and procollagen [17-19]. Additionally, there may be a considerable increase in collagen bundles and, in some cases, deposits of mucin in the skin [20,21]. Microscopic changes similar to those described in human NSF patients were observed in the rats showing macroscopic skin changes (only animals treated with Omniscan). The development of fibrosis, including increased cellularity and increased cell swelling causing a loss of interstitial space was observed in five out of six animals treated with Omniscan. Furthermore, these changes were accompanied by an increased dermal cell count. We also found an increase in the number of CD34positive cells in the dermis of those animals showing fibrosis [8]. It is important to note, however, that we observed differences in the histopathology between that reported in human NSF patients and the rats used in our experimental setting. For example, in the rats, we did not see the deep infiltration of the sub-cutis found in human NSF patients. It is possible that this difference is due to the different histology of the skin in rats [8].

The occurrence of these skin lesions seems to be strongly correlated with high Gd concentrations, particularly in the skin, but also in the liver and the femur, confirming the previous finding [8], which suggests that there may be a relationship between the release of $\mathrm{Gd}$ and the occurrence of the NSF-like lesions.

Stability/inertness characteristics measured in vitro describe the likelihood of Gd release from the Gd-chelate in vitro, which may be a predictor for release of Gd ions in vivo. In our setting we were able to test whether in vitro stability data correlate with Gd concentrations in the skin of the investigated animals.

Regarding their in vitro stability, Gd-containing contrast agents fall into two distinct classes: the linear, open-chain agents and the macrocyclic agents [22]. 
Table 3 Relevant stability parameters [25] of the investigated linear chelates

\begin{tabular}{lllll}
\hline Contrast agent & Charge & $\begin{array}{l}\text { Thermodynamic stability } \\
\left(\log \mathrm{K}_{\text {therm }}, \mathrm{pH} \mathrm{14}\right)\end{array}$ & $\begin{array}{l}\text { Conditional stability } \\
\left(\log \mathrm{K}_{\text {cond }}, \mathrm{pH} 7.4\right)\end{array}$ & $\begin{array}{l}\text { Excess free ligand } \\
\text { in formulation }(\mathrm{mg} / \mathrm{ml})\end{array}$ \\
\hline Omniscan & Non-ionic & 16.9 & 14.9 & 12 \\
OptiMARK & Non-ionic & 16.6 & 15.0 & 28.4 \\
Magnevist & Ionic & 22.5 & 18.4 & 0.4 \\
MultiHance & Ionic & 22.6 & 18.4 & $0.4[30]^{\mathrm{a}}$ \\
\hline
\end{tabular}

${ }^{\mathrm{a}}$ Recent information suggests that in the presently marketed formulation of MultiHance no excess ligand is contained

Linear complexes differ significantly in their stability, which may be adequately described by the thermodynamic stability constant $\left(\log \mathrm{K}_{\text {therm, }}\right.$, valid at $\left.\mathrm{pH}=14\right)$ and the conditional stability constant $\log \mathrm{K}_{\text {cond }}$ (calculated for $\mathrm{pH}=7.4$ ). The addition of an ionic charge enhances the stability of the complex, with the ionic linear complexes (e.g. Magnevist and MultiHance) being about 3.5 orders of magnitude (or approximately a factor of 3,000) more stable than the non-ionic linear complexes (Omniscan and OptiMARK) (Table 3, Fig. 7a). To compensate for their lower stability, the non-ionic linear compounds, Omniscan and OptiMark, are formulated with $5 \mathrm{~mol} \%$ and $10 \mathrm{~mol} \%$ excess ligand, respectively [23].

In vitro data demonstrate that macrocyclic Gd chelates are kinetically inert to $\mathrm{Gd}$ dissociation compared to linear Gd chelates [24-26] since a substantial activation energy barrier has to be overcome for both the complexation and the de-complexation of marketed macrocyclic agents (Fig. 7b). The reason for this phenomenon is the fact that the macrocyclic ring system is very rigid and additional energy is needed to set the complexed gadolinium free
Fig. 7 Differences in stability/ inertness of gadoliniumcontaining contrast agents. The differences between non-ionic and ionic linear agents (a) and between linear and macrocyclic agents (b) are depicted a

\section{Linear agents}

Ionic

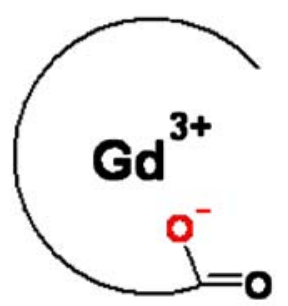

stronger

b

linear, open chain complexes kinetically less stable, thermodynamic stability
overriding

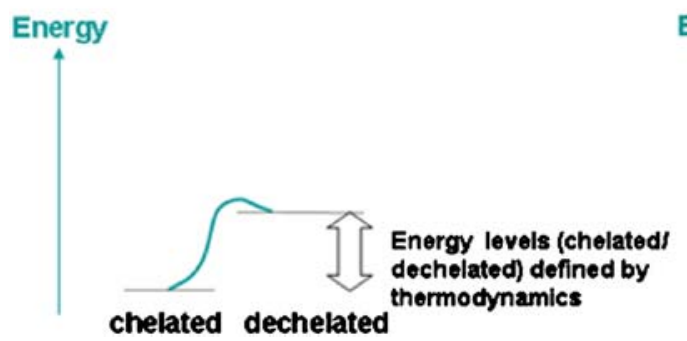

Non-ionic

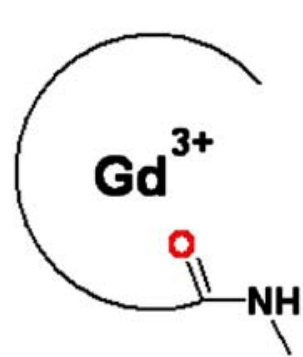

weaker

macrocyclic complexes

Kinetic inertness overriding

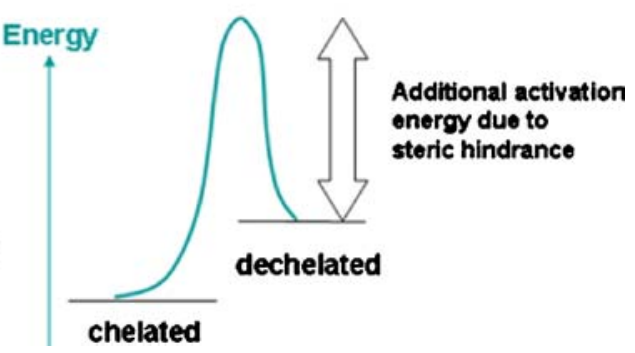


Table 4 Relevant stability parameters [25] of the investigated macrocyclic chelates

\begin{tabular}{lllll}
\hline Contrast agent & Charge & $\begin{array}{l}\text { Dissociation rate } \mathrm{k}_{\mathrm{obs}} \\
\left(\mathrm{s}^{-1}\right)(\mathrm{pH} \mathrm{1.0)}\end{array}$ & $\begin{array}{l}\text { Dissociation half-life } \\
\left(\mathrm{T}_{1 / 2} \text { at } \mathrm{pH} \mathrm{1.0,} \mathrm{calculated} \mathrm{from} \mathrm{k}_{\mathrm{obs}}\right)\end{array}$ & $\begin{array}{l}\text { Dissociation half-life } \\
\left(\mathrm{T}_{1 / 2}, \text { extrapolated to } \mathrm{pH} 7.4\right)\end{array}$ \\
\hline \multirow{2}{*}{ Dotarem } & Ionic & $2.1 \times 10^{-5}$ & $9 \mathrm{~h}$ & $>1,000$ years \\
& & $3.2 \times 10^{-6}$ & $60 \mathrm{~h}$ & $>1,000$ years \\
Gadovist & Non-ionic & $8.0 \times 10^{-6}$ & $24 \mathrm{~h}$ & \\
\hline
\end{tabular}

from the sterically hindered (bulky) macrocycle. Therefore the propensity of macrocylic GBCAs to release gadolinium is best described by the kinetic constant $\left(\mathrm{k}_{\mathrm{obs}}\right.$, measured at $\mathrm{pH}=1.0$ and derived from the dissociation half-life) and not by the thermodynamic stability constants $\log \mathrm{K}_{\text {therm }}$ and $\log \mathrm{K}_{\text {cond }}$ (Table 4) [25].

In our experimental setting, Omniscan exposure resulted in the highest $\mathrm{Gd}$ concentrations of all tested agents, especially in the skin, followed by OptiMARK. As the stability constants of the two agents are similar, this suggests that the difference in Gd concentration is caused by the different amount of excess ligand. We were able to confirm this role of the excess ligand in a separate series of experiments more thoroughly [27].

Significantly smaller Gd concentrations were measured in the animals treated with the ionic linear agents Magnevist and MultiHance compared to both non-ionic linear agents. As described above, ionic linear agents are characterized by a higher in vitro stability as compared to non-ionic linear agents. The difference in the Gd concentration observed between MultiHance- and Magnevisttreated animals can, however, not be easily explained by differences in in vitro stabilities, as those were very similar (see Table 3). The observation can most likely be explained by the different exposure of the animals to the contrast agents. MultiHance is excreted up to $50 \%$ via the hepatobiliary system in rats [28] causing faster excretion and thereby a lower AUC. This difference compared to the other GBCAs is not seen in humans, where the hepatobiliary excretion of MultiHance accounts only for $0.6-4 \%$ [29]. Therefore, the observations regarding the Gd concentration after treatment with MultiHance in the rat model may not be an accurate predictor of the clinical situation.

The lowest Gd concentrations, significantly lower than the Gd concentrations of all linear agents with similar pharmacokinetic clearance, were measured in the animals treated with the macrocyclic Gd chelates Gadovist and Dotarem. Although the kinetic half-lifes measured in vitro at $\mathrm{pH} 1.0$ for Gadovist and Dotarem were not identical (see Table 4), the present investigation found virtually identical, low levels of $\mathrm{Gd}$ in the skin. This finding suggests that there are no relevant stability differences of the macrocyclic compounds in vivo as already postulated based on the very long extrapolated dissociation half-life in vitro at $\mathrm{pH} 7.4$ [25].
Given their high stability, it may be expected that no Gd would be detectable in animals treated with the two tested macrocyclic agents, Gadovist and Dotarem. However, in our experimental setting, Gd was detected with both agents, albeit at significantly lower amounts. The residual Gd could be related to the specifics of the ICP-AES method, which can not distinguish between chelated and unchelated gadolinium. It is possible that some or all of the residual Gd measured in the tissue specimens taken 5 days after the last injection arises from chelated $\mathrm{Gd}$, which will not be retained in the skin but eliminated over time. Of course, the same caveat applies to the Gd concentrations measured after the applications of all other agents in the study. However, we did demonstrate in our first experiment that unchelated $\mathrm{Gd}$ is present in precipitates in the skin of animals treated with Omniscan [8]. Studies to measure the time course of Gd retention in the skin are currently being conducted, but results are not yet available.

To summarize the data obtained in this investigation (given the limitations of the experimental setting), nonionic linear Omniscan seems clearly to be the agent with the highest risk of releasing $\mathrm{Gd}$ ions in vivo (the situation is slightly improved with the addition of more excess ligand, as in OptiMARK), while the risk is significantly reduced with ionic linear agents, such as Magnevist and MultiHance, and even further reduced with macrocyclic agents, such as Gadovist and Dotarem.

Although in our preclinical setting NSF-like skin lesions were noted to occur only in the Omniscan treatment group, cases of NSF reported to date in humans have been associated with the use, not only of Omniscan, but also of other GBCAs as well (for latest information refer to the websites of health authorities such as the FDA). Furthermore, since residual Gd concentrations were found in this experiment for the non-Omniscan animals, the risk for NSF can not be excluded for the other agents.

In conclusion, our preclinical experiments suggest that the different in vitro stabilities of the Gd-containing contrast agents result in different propensities to release Gd, particularly in the skin. The findings of this investigation support the conclusion that the complex stability of GBCAs is correlated with the likelihood of releasing Gd ions in vivo. It remains to be seen whether these findings correlate with a different risk of NSF. 
Acknowledgements The authors would like to thank Leigh Prevost, BSc Pharm, Medicus International, for his editorial assistance. The authors would like to thank Christiane Pering, M.D., Daniel Grosu, M.D., and Andrea Löwe, M.D., for the critical reading of the manuscript and Ines Heinzelmann, Stefanie Runge, and Andrea Baumgart for their excellent technical assistance.
Open Access This article is distributed under the terms of the Creative Commons Attribution Noncommercial License which permits any noncommercial use, distribution, and reproduction in any medium, provided the original author(s) and source are credited.

\section{References}

1. Broome DR, Girguis MS, Baron PW, Cottrell AC, Kjellin I, Kirk GA (2007) Gadodiamide-associated nephrogenic systemic fibrosis: why radiologists should be concerned. AJR Am J Roentgenol 188:586-592

2. Cowper SE, Robin HS, Steinberg SM, Su LD, Gupta S, LeBoit PE (2000) Scleromyxoedema-like cutaneous diseases in renal-dialysis patients. Lancet 356:1000-1001

3 . US FDA (2007) Information on gadolinium-containing contrast agents. http://www.fda.gov/cder/drug/infopage/ gcca/. Accessed 7 April 2008

4. Saab G, Abu-Alfa A, Sadowski EA, Djamali A (2007) Are patients with moderate renal failure at risk for developing nephrogenic systemic fibrosis? Radiology 244:930-932

5. DeHoratius DM, Cowper SE (2006) Nephrogenic systemic fibrosis: an emerging threat among renal patients. Semin Dial 19:191-194

6. Grobner T (2006) Gadolinium-a specific trigger for the development of nephrogenic fibrosing dermopathy and nephrogenic systemic fibrosis? Nephrol Dial Transplant 21:3604-3605

7. Marckmann P, Skov L, Rossen K, Dupont A, Damholt MB, Heaf JG, Thomsen HS (2006) Nephrogenic systemic fibrosis: suspected causative role of gadodiamide used for contrastenhanced magnetic resonance imaging. J Am Soc Nephrol 17:2359-2362

8. Sieber MA, Pietsch H, Walter J, Haider W, Frenzel T, Weinmann H-J (2008) A preclinical study to investigate the development of nephrogenic systemic fibrosis: a possible role for gadoliniumbased contrast media. Invest Radiol 43:65-75

9. Collidge TA, Thomson PC, Mark PB, Traynor JP, Jardine AG, Morris ST, Simpson K, Roditi GH (2007) Gadolinium-enhanced MR imaging and nephrogenic systemic fibrosis: retrospective study of a renal replacement therapy cohort. Radiology 245:168-175

10. Wahba IM, Simpson EL, White K (2007) Gadolinium is not the only trigger for nephrogenic systemic fibrosis: insights from two cases and review of the recent literature. Am J Transplant 7:2425-2432
11. Thomsen HS, Morcos SK, Dawson P (2006) Is there a causal relation between the administration of gadolinium based contrast media and the development of nephrogenic systemic fibrosis (NSF)? Clin Radiol 61:905-906

12. Crissman JW, Goodman DG, Hildebrandt PK, Maronpot RR, Prater DA, Riley JH, Seaman WJ, Thake DC (2004) Best practices guideline: toxicologic histopathology. Toxicol Pathol 32:126-131

13. Greaves P (2000) Integumentary system. Elsevier, Amsterdam

14. Klein-Szanto A, Conti C (2002) Skin and oral mucosa. Academic Press, San Diego

15. Noelte J (2002) ICP Emissionsspektometrie für Praktiker. Wiley-VCH, Weinheim

16. Sadowski EA, Bennett LK, Chan MR, Wentland AL, Garrett AL, Garrett RW, Djamali A (2007) Nephrogenic systemic fibrosis: risk factors and incidence estimation. Radiology 243:148-157

17. Cowper SE, Bucala R (2003) Nephrogenic fibrosing dermopathy: suspect identified, motive unclear. Am J Dermatopathol 25:358

18. Ortonne N, Lipsker D, Chantrel F, Boehm N, Grosshans E, Cribier B (2004) Presence of CD45RO+ CD34+ cells with collagen synthesis activity in nephrogenic fibrosing dermopathy: a new pathogenic hypothesis. Br J Dermatol 150: $1050-1052$

19. Kucher C, Xu X, Pasha T, Elenitsas R (2005) Histopathologic comparison of nephrogenic fibrosing dermopathy and scleromyxedema. J Cutan Pathol 32:484-490

20. Edsall LC, English JC 3rd, Teague MW, Patterson JW (2004) Calciphylaxis and metastatic calcification associated with nephrogenic fibrosing dermopathy. J Cutan Pathol 31:247253

21. Lewis KG, Lester BW, Pan TD, Robinson-Bostom L (2006) Nephrogenic fibrosing dermopathy and calciphylaxis with pseudoxanthoma elasticum-like changes. J Cutan Pathol 33:695-700
22. Caravan P, Ellison JJ, McMurry TJ, Lauffer RB (1999) Gadolinium(III) chelates as MRI contrast agents: structure, dynamics, and applications. Chem Rev 99:2293-2352

23. Harpur ES, Worah D, Hals PA, Holtz E, Furuhama K, Nomura H (1993) Preclinical safety assessment and pharmacokinetics of gadodiamide injection, a new magnetic resonance imaging contrast agent. Invest Radiol 28(Suppl 1):S28-S43

24. Idee JM, Port M, Raynal I, Schaefer M, Le Greneur S, Corot C (2006) Clinical and biological consequences of transmetallation induced by contrast agents for magnetic resonance imaging: a review. Fundam Clin Pharmacol 20:563-576

25. Schmitt-Willich H (2007) Stability of linear and macrocyclic gadolinium based contrast agents. Br J Radiol 80:581-582, author reply 584-585

26. Tweedle MF (2007) "Stability" of gadolinium chelates. Br J Radiol 80:583584, author reply 584-585

27. Sieber MA, Lengsfeld P, Walter J, Schirmer H, Frenzel T, Siegmund F, Weinmann HJ, Pietsch H (2008) Gadolinium-based contrast agents and their potential role in the pathogenesis of nephrogenic systemic fibrosis: the role of excess ligand. J Magn Reson Imaging 27:955-962

28. Planchamp C, Pastor CM, Balant L, Becker CD, Terrier F, Gex-Fabry M (2005) Quantification of Gd-BOPTA uptake and biliary excretion from dynamic magnetic resonance imaging in rat livers: model validation with $153 \mathrm{Gd}$ BOPTA. Invest Radiol 40:705-714

29. Spinazzi A, Lorusso V, Pirovano G, Taroni P, Kirchin M, Davies A (1998) Multihance clinical pharmacology: biodistribution and MR enhancement of the liver. Acad Radiol 5(Suppl 1): S86-S89, discussion S93-84

30. Shellock FG, Parker JR, Pirovano G, Shen N, Venetianer C, Kirchin MA, Spinazzi A (2006) Safety characteristics of gadobenate dimeglumine: clinical experience from intra- and interindividual comparison studies with gadopentetate dimeglumine. J Magn Reson Imaging 24:1378-1385 